\title{
Green's Impedance Function Approach for Propagation Characteristics of Generalized Striplines and Slotlines on Nonlayered Substrates
}

\author{
Jeng-Wen Huang, Student Member, IEEE, and Ching-Kuang C. Tzuang, Senior Member, IEEE
}

\begin{abstract}
A newly proposed generalized full-wave spacedomain integral equation technique incorporating dyadic Green's impedance function and a new set of basis functions are presented for the analysis of striplines and slotlines arbitrarily integrated on multiple nonlayered sustrates. The generalized full-wave integral equation technique is validated by conducting convergence study and several case studies. The theoretical dispersion characteristic of the transmission lines obtained here are in very good agreement with the published and measured data for the modified Microslab and the microstrip proximity effect near substrate edge. For the slotline type problem, where a new set of current basis function must be derived to be incorperated into Green's impendance function approach, the new approach presented here shows that the corresponding propagation characteristics thus obtained are also in excellent agreement with those reported in the available literature, which normally used the slot electric fields as the unknowns. To explain how general the present formulation is, it can be reduced to the well-known spectral domain formulation for any guided-wave structure containing only layered substrates.
\end{abstract}

\section{INTRODUCTION}

$\mathrm{N}$ the past two decades guided-wave structures consisting of layered and nonlayered substrates, the latter having step discontinuities in the dielectric constant of layers, have been studied characterized both theoretically and experimentally for various applications in electrooptic modulators [1]-[3], low-loss millmeter-wave transmission lines [4], [5], the mode compensation technique on a pair of parallel-coupled microstrip lines [6]-[8], high- $Q$ MMIC (monolithic microwave integrated circuits) resonators [9], and resonant phenomena in MMIC packaging [10], etc. In view of the forementioned applications for MMIC a system of transmission lines can be pictured in Fig. 1, where two dominant features may convert Fig. 1 structure to virtually almost any planar or quasi-planar transmission line configuration known to date.

First, each layer has step discontinuities in dielectric constant for $y=d_{k}, k=1,2, \ldots, N-1$. By setting the thickness values of appropriate layers equal to zero, complex structures such as ridge substrate, microslab waveguide, microstrip (or slot line) and dielectric waveguide, and slot line of recessed gap can be realized easily.

Manuscript received October 14, 1993; revised February 8, 1994. This work was supported by the National Science Council, Republic of China, under Grant NSC 82-0404-D009-033 and NSC83-0404-E009-045.

The authors are with the Institute of Communication Engineering, National Chiao Tung University, Hsinchu, Taiwan, R.O.C.

IEEE Log Number 9405370 .

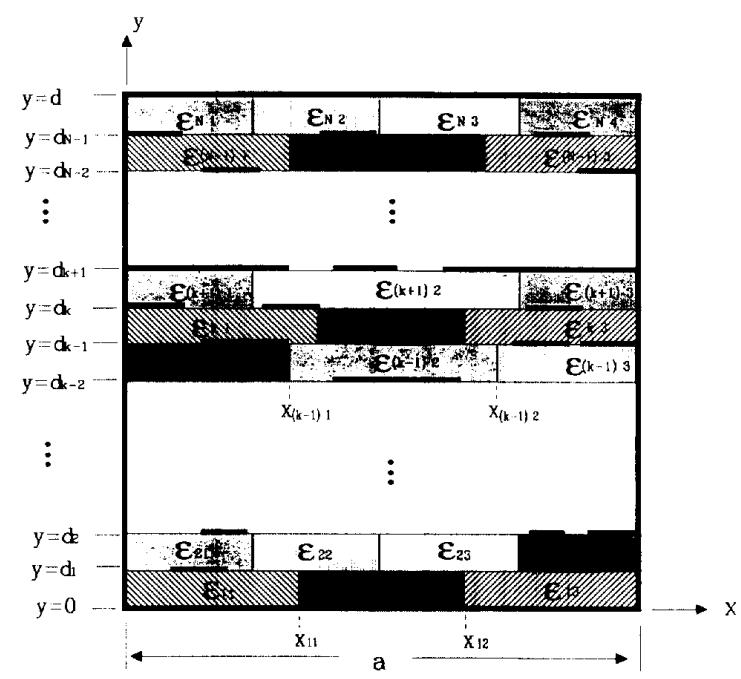

Fig. 1. A generic system of striplines and slotlines on multiple nonlayered substrates for use in microwave, millimeter-wave, and electrooptic circuit designs. The metal strips are assumed infinitely thin and perfect conductors.

Second, each layer consists of randomly-distributed strips and/or side planes connected to the electrically shielded sidewalls. If two side planes and one central coplanar strip are incorporated, it will look like a coplanar waveguide (cpw) structure. Various arrangements of microstrips and side planes lead to a variety of transmission line structures. Notice that both slotline type and microstrip type guided structures are inherently mingled in Fig. 1.

The full-wave analysis of propagation characteristics of Fig. 1 is necessary for most circumstances when carrying out a design project related to the above-mentioned applications and is by no means a simple task. Generally speaking three categories of numerical solutions can be invoked to tackle the problem. At one end, FEM (finite-elementmethod) [11], [12], finite-difference in frequency [13], [14] and time-domain [15], [16] may analyze the guidedwave structure of arbitrary shape; however, they normally require large memory space in the program code to achieve desired accuracy. On the other hand, the spectral-domain approach or space-domain integral equation approach [17] or mode-matching method [18] uses comparatively less 
memory and CPU time to achieve desired accuracy of the guided-wave propagation characteristics at the expanse of somewhat lengthy derivations for dyadic Green's functions which are limited to structures with layered or stratified substrates [19]-[21] except for very few examples that both layered and nonlayered substrates of step-discontinuity in dielectric constant can be considered simultaneously [22], [23]. Between the two extremes, the method of lines had been reported for the reduced guided-wave structures of Fig. 1 [24], [25].

When considering a guided structure where both slotlines and striplines coexist, the well-known Spectral Domain Immitance Approach (SDIA) is numerically efficient and very accurate and thus widely adopted for obtaining the propagation characteristics of the transmission line system embedded in the layered substrates [20]. As shown in Fig. 1, the guided-structure is nonlayered; thereby a modified approach is required to obtain the dyadic Green's function. Section II shows how the new dyadic Green's impedance function is obtained for Fig. 1. The unknowns in the new dyadic Green's impedance function are all in terms of surface currents on the metal strips regardless of whether the striplines or slotlines are under investigation. Section III develops a new set of basis functions for slotlines problems where two side planes may be connected to side-walls. Combined use of the new basis functions and those developed previously for striplines problem [26], the propagation characteristics of Fig. 1 can be solved very accurately.

Followed by the validity check of the present approach against existing literature [22], [23], [25] in Section IV and the convergence study are also conducted. Section V concludes the paper.

\section{Formulation DyAdic GREEN'S IMPEDANCE FUNCTION OF THE SLOTLINES AND STRIPLINES IN NONLAYERED SUBSTRATES}

In Fig. 1, metal strips are assumed infinitely thin perfect conductors surrounded by rectangular perfect electric walls. Assume that the time-harmonic dependence is $e^{j \omega t}$ and guided-wave $z$-dependant solution is $e^{-j \gamma z}$. Omitting the $e^{j \omega t}$ time-dependant term in the formulation, the point-source current density can be written as

$$
\begin{aligned}
\widetilde{J}\left(x, y ; x^{\prime}, y^{\prime}\right)= & J_{z} \delta\left(x-x^{\prime}\right) \delta\left(y-y^{\prime}\right) e^{-j \gamma z} \hat{z} \\
& +J_{x} \delta\left(x-x^{\prime}\right) \delta\left(y-y^{\prime}\right) e^{-j \gamma z} \hat{x}
\end{aligned}
$$

where $J_{z}$ and $J_{x}$ represent the longitudinal and transverse current components, respectively; $\hat{x}$ and $\hat{z}$ are the unity constant vector in the $x$ and $z$ direction, respectively.

The first step in deriving the dyadic Green's impedance function is to obtain the correct eigenfunction expansions in nonlayered substrate region such that the boundary conditions are satisfied at the step discontinuities at the dielectric interface and at the enclosure [22], [23], [27], [28]. In an arbitrary $k$ th source-free region (See Fig. 1), $d_{k-1} \leq y \leq d_{k}$, the electromagnetic fields satisfying the Helmholtz equation can be expressed as

$$
\begin{aligned}
& \bar{E}^{(k)}=\nabla \times \nabla \times \bar{\pi}_{e}^{(k)}-j \omega \mu \nabla \times \bar{\pi}_{h}^{(k)}, \\
& \bar{H}^{(k)}=j \omega \varepsilon \nabla \times \bar{\pi}_{\mathrm{e}}^{(k)}+\nabla \times \nabla \times \bar{\pi}_{h}^{(k)} .
\end{aligned}
$$

Since the solutions for the Helmholtz equation are separable in Cartesian coordinates, the hybrid TE-to- $x$ and TM-to- $x$ Hertzian potentials in (2a) and (2b) can be written as

$$
\begin{aligned}
& \bar{\pi}_{e}^{(k)}(x, y, z)=\sum_{m=1}^{M} \phi_{m}^{e(k)}(x) \psi_{m}^{e(k)}(y) e^{-j \gamma z} \hat{x} \\
& \bar{\pi}_{h}^{(k)}(x, y, z)=\sum_{m=1}^{M} \phi_{m}^{h(k)}(x) \psi_{m}^{h(k)}(y) e^{-j \gamma z} \hat{x}, \text { respectively, }
\end{aligned}
$$

where $k$ denotes the index from region 1 to $N$ and $M$ denotes the total number of each eigenfunction expansion terms. The normalized Hertzian potential functions $\psi_{m}^{e(k)}(y)$ and $\psi_{m}^{h(k)}(y)$ can be expressed as

$$
\begin{aligned}
\psi_{m}^{e(k)}(y)= & a_{m}^{e(k)} \frac{\sin \left[\beta_{y_{m}}^{e(k)}\left(y-y_{k}\right)\right]}{\sin \left[\beta_{y_{m}}^{e(k)} y_{k}^{\prime}\right]} \\
& +b_{m}^{e(k)} \frac{\cos \left[\beta_{y_{m}}^{e(k)}\left(y-y_{k}\right)\right]}{\cos \left[\beta_{y_{m}}^{e(k)} y_{k}^{\prime}\right]} \\
\psi_{m}^{h(k)}(y)= & a_{m}^{h(k)} \frac{\cos \left[\beta_{y_{m}}^{h(k)}\left[y-y_{k}\right)\right]}{\cos \left[\beta_{y_{m}}^{h(k)} y_{k}^{\prime}\right]} \\
& +b_{m}^{h(k)} \frac{\sin \left[\beta_{y_{m}}^{h(k)}\left[y-y_{k}\right)\right]}{\sin \left[\beta_{y_{m}}^{h(k)} y_{k}^{\prime}\right]}
\end{aligned}
$$

and the corresponding potential functions $\phi_{m}^{e(k)}(x)$ and $\phi_{m}^{h(k)}(x)$ can be expressed as

$$
\begin{aligned}
\phi_{m}^{e(k)}(x)= & c_{m}^{e\left(k_{i}\right)} \cos \left[\beta_{x_{m}}^{e\left(k_{i}\right)}\left(x-x_{k_{i}}\right)\right] \\
& +d_{m}^{e\left(k_{i}\right)} \sin \left[\beta_{x_{m}}^{e\left(k_{i}\right)}\left(x-x_{k_{i}}\right)\right], \\
\phi_{m}^{h(k)}(x)= & c_{m}^{h\left(k_{i}\right)} \cos \left[\beta_{x_{m}}^{h_{(}\left(k_{i}\right)}\left(x-x_{k_{i}}\right)\right] \\
& +d_{m}^{h\left(k_{i}\right)} \sin \left[\beta_{x_{m}}^{h\left(k_{i}\right)}\left(x-x_{k_{i}}\right)\right]
\end{aligned}
$$

where

$$
\begin{aligned}
\left(\beta_{y_{m}}^{e(k)}\right)^{2} & =\varepsilon_{r}^{(k)}(x) k_{0}^{2}-\left(\beta_{x_{m}}^{e\left(k_{i}\right)}\right)^{2}-\gamma^{2} \\
\left(\beta_{y_{m}}^{h(k)}\right)^{2} & =\varepsilon_{r}^{(k)}(x) k_{0}^{2}-\left(\beta_{x_{m}}^{h\left(k_{i}\right)}\right)^{2}-\gamma^{2}, \quad\left(k_{0}^{2}=\omega^{2} \mu_{0} \varepsilon_{0}\right) \\
y_{k}^{\prime} & =\left[d_{k}-d_{k-1}\right] / 2 \\
y_{k} & =\left[d_{k}+d_{k-1}\right] / 2
\end{aligned}
$$

The determination of the unknown coefficients $c_{m}^{e\left(k_{i}\right)}, d_{m}^{e\left(k_{i}\right)}$, $c_{m}^{h\left(k_{i}\right)}$, and $d_{m}^{h\left(k_{i}\right)}$ and the eigenvalues $\beta_{x m}^{e(k i)}$ and $\beta_{x m}^{h(k i)}$ of (5a) and (5b) is straightforward when the tangential boundary conditions at $x=x_{k i}, i=1, \ldots, p_{k}$ [28], where $p_{k}$ is number of dielectric step discontinuity of the $k$ th region in $x$-direction. The remaining $a_{m}^{e(k)}, b_{m}^{e(k)}, a_{m}^{h(k)}$, and 
$b_{m}^{h(k)}$ coefficients constitute four sets of unknown coefficients to be determined. Notice that in region 1 and region $N$, only two sets of coefficients i.e., $a_{m}^{e(k)}$ and $a_{m}^{h(k)}$ are necessary.

Substituting equations (4) and (5) into (3) and invoking (2a) and $(2 b)$, one obtains the total field in region $k$

$$
\begin{aligned}
E_{x}^{(k)}= & \sum_{m=1}^{M}\left[\left(\beta_{y_{m}}^{e(k)}\right)^{2}+\gamma^{2}\right] \frac{\phi_{m}^{e(k)}(x)}{\varepsilon_{r}^{(k)}(x)} \psi_{m}^{e(k)}(y) e^{-j \gamma z}, \\
H_{x}^{(k)}= & \sum_{m=1}^{M}\left[\left(\beta_{y_{m}}^{h(k)}\right)^{2}+\gamma^{2}\right] \phi_{m}^{h(k)}(x) \psi_{m}^{h(k)}(y) e^{-j \gamma z}, \\
E_{y}^{(k)}= & \sum_{m=1}^{M} \frac{1}{\varepsilon_{r}^{(k)}(x)} \frac{\partial \phi_{m}^{e(k)}(x)}{\partial x} \frac{\partial \psi_{m}^{e(k)}(y)}{\partial y} e^{-j \gamma z} \\
& +\sum_{m=1}^{M}\left(-\omega \mu_{0} \gamma\right) \phi_{m}^{h(k)}(x) \psi_{m}^{h(k)}(y) e^{-j \gamma z} \\
H_{y}^{(k)}= & \sum_{m=1}^{M}\left(\omega \gamma \varepsilon_{0}\right) \phi_{m}^{e(k)}(x) \psi_{m}^{e(k)}(y) e^{-j \gamma z} \\
& +\sum_{m=1}^{M} \frac{\partial \phi_{m}^{h(k)}(x)}{\partial x} \frac{\partial \psi_{m}^{h(k)}(y)}{\partial y} e^{-j \gamma z}, \\
E_{z}^{(k)}= & \sum_{m=1}^{M}(-j \gamma) \frac{1}{\varepsilon_{r}^{(k)}(x)} \frac{\partial \phi_{m}^{e(k)}(x)}{\partial x} \psi_{m}^{e(k)}(y) e^{-j \gamma z} \\
& +\sum_{m=1}^{M}\left(j \omega \mu_{0}\right) \phi_{m}^{h(k)}(x) \frac{\partial \psi_{m}^{h(k)}(y)}{\partial y} e^{-j \gamma z} \\
& +\sum_{m=1}^{M}(-j \gamma) \frac{\partial \phi_{m}^{h(k)}(x)}{\partial x} \psi_{m}^{h(k)}(y) e^{-j \gamma z} \\
H_{z}^{(k)}= & \sum_{m=1}^{M}\left(-j \omega \varepsilon_{0}\right) \phi_{m}^{e(k)}(x) \frac{\partial \psi_{m}^{e(k)}(y)}{\partial y} e^{-j \gamma z} \\
& \sum_{m}(6)
\end{aligned}
$$

At $y=d_{k}$, for example, the boundary condition reads as

$$
\begin{aligned}
& E_{z}^{(k+1)}(x)-E_{z}^{(k)}(x)=0, \quad(0 \leq x \leq a) \\
& H_{z}^{(k+1)}(x)-H_{z}^{(k)}(x)=J_{x}^{(k)} \delta\left(x-x^{\prime}\right), \\
& \quad(0 \leq x \leq a), x^{\prime} \in \text { strips, } \\
& E_{x}^{(k+1)}(x)-E_{x}^{(k)}(x)=0, \quad(0 \leq x \leq a) \\
& H_{x}^{(k)}(x)-H_{x}^{(k+1)}(x)=J_{z}^{(k)} \delta\left(x-x^{\prime}\right), \\
& \quad(0 \leq x \leq a), x^{\prime} \in \text { strips, } \\
& E_{z}^{(k+1)}(x)=E_{z}^{(k)}(x)=0, \quad x \in \text { strips, } \\
& E_{x}^{(k+1)}(x)=E_{x}^{(k)}(x)=0, \quad x \in \text { strips. }
\end{aligned}
$$

Notice that the $x$-directed eigenfunctions satisfy the wellknown biorthogonality relations

$$
\begin{aligned}
& \int_{0}^{a} \frac{\phi_{n}^{e(k)}(x) \phi_{m}^{e(k)}(x)}{\varepsilon_{r}^{(k)}(x)} d x=\delta_{m n}^{e} \\
& \int_{0}^{a} \phi_{n}^{h(k)}(x) \phi_{m}^{h(k)}(x) d x=\delta_{m n}^{h},
\end{aligned}
$$

where $\delta_{m n}^{e}$ and $\delta_{m n}^{h}$ are the Kronecker delta functions.
When one considers the interface matching at $y=d_{k}$ and $y=d_{k-1}$, respectively, the following matrix equations can be obtained by substituting the corresponding tangential field components as shown in (6) into 7(a)-7(d) and applying the biorthogonality relations equations (8a) and (8b).

$$
\begin{gathered}
{\left[\begin{array}{c}
a_{m}^{e(k)} \\
b_{m}^{e(k)} \\
a_{m}^{h(k)} \\
b_{m}^{h(k)}
\end{array}\right]=} \\
{\left[V^{(k, k+1)}\right]_{4 M \times 4 M}\left[\begin{array}{l}
a_{m}^{e(k+1)} \\
b_{m}^{e(k+1)} \\
a_{m}^{h(k+1)} \\
b_{m}^{h(k+1)}
\end{array}\right]} \\
+\left[I^{(k, k+1)}\right]_{4 M \times 2 M}\left[\begin{array}{l}
J_{x m}^{(k)}\left(x^{\prime}\right) \\
J_{z m}^{(k)}\left(x^{\prime}\right)
\end{array}\right], \\
\text { at } y=d_{k} \text { for } m=1,2, \ldots, M
\end{gathered}
$$

and

$$
\begin{array}{ll}
J_{x m}^{(i)}\left(x^{\prime}\right)=J_{x}^{(i)} \frac{\phi_{m}^{e(j)}\left(x^{\prime}\right)}{\varepsilon_{r}^{(j)}\left(x^{\prime}\right)}, & i=k-1, k ; j=k, \\
& \text { for } m=1,2, \ldots, M(10 \mathrm{a}) \\
J_{z m}^{(i)}\left(x^{\prime}\right)=J_{z}^{(i)} \phi_{m}^{h(j)}\left(x^{\prime}\right), & i=k-1, k ; j=k, \\
& \text { for } m=1,2, \ldots, M(10 \mathrm{~b})
\end{array}
$$

where $\left[V^{(k, k+1)}\right]_{4 M \times 4 M}$ and $\left[\Lambda^{(k, k-1)}\right]_{4 M \times 4 M}$ are called the upper and lower chain matrix relating the corresponding adjacent set of coefficients respectively; whereas $\left[I^{(k, k+1)}\right]_{4 M \times 2 M}$ and $\left[I^{(k, k-1)}\right]_{4 M \times 2 M}$ are called the associated current matrices relating the current densities to the unknown coefficients. For the sake of clarity and completeness, the elements of the matrices shown in (9a) and (9b) are listed in Appendix A. Notice that if there is no strip existing at certain interface, the corresponding current matrix $\left[I^{(k, k+1)}\right]_{4 M \times 2 M}$ and $\left[I^{(k, k-1)}\right]_{4 M \times 2 M}$ will become null matrices.

Referring to the structure in Fig. 1 and by applying (9a) and $(9 \mathrm{~b})$ recursively, one can obtain the following equations relating the coefficient vector at an arbitrarily selected layer at $y \in\left(d_{k-1}, d_{k}\right)$ to the coefficient vectors at the top and bottom layers as well as all the current densities of the strips

$$
\begin{aligned}
{\left[\begin{array}{c}
a_{m}^{e(k)} \\
b_{m}^{e(k)} \\
a_{m}^{h(k)} \\
b_{m}^{h(k)}
\end{array}\right]=} & \prod_{i=k}^{N-1}\left[V^{(i, i+1)}\right]_{4 M \times 2 M}\left[\begin{array}{c}
a_{m}^{e(N)} \\
a_{m}^{h(N)}
\end{array}\right] \\
& +\left[I^{(k, k+1)}\right]_{4 M \times 2 M}\left[\begin{array}{c}
J_{x m}^{(k)}\left(x^{\prime}\right) \\
J_{z m}^{(k)}\left(x^{\prime}\right)
\end{array}\right] \\
& +\sum_{j=k+1}^{N-1} \prod_{i=k+1}^{j}\left[V^{(i-1, i)}\right]\left[I^{(j, j+1)}\right]\left[\begin{array}{l}
J_{x m}^{(j)}\left(x^{\prime}\right) \\
J_{z m}^{(j)}\left(x^{\prime}\right)
\end{array}\right],
\end{aligned}
$$




$$
\begin{aligned}
{\left[\begin{array}{l}
a_{m}^{e(k)} \\
b_{m}^{e(k)} \\
h(k) \\
a_{m} \\
b_{m}^{h(k)}
\end{array}\right]=} & \prod_{i=1}^{k-1}\left[\Lambda^{(k+1-i, k-i)}\right]_{4 M \times 2 M}\left[\begin{array}{l}
a_{m}^{e(1)} \\
a_{m}^{(1)}
\end{array}\right] \\
+ & {\left[I^{(k, k-1)}\right]_{4 M \times 2 M}\left[\begin{array}{l}
J_{x m}^{(k-1)}\left(x^{\prime}\right) \\
J_{z m}^{(k-1)}\left(x^{\prime}\right)
\end{array}\right] } \\
& +\sum_{j=2}^{k-1} \prod_{i=1}^{j-1}\left[\Lambda^{(k+1-i, k-i)}\right] \\
& \times\left[I^{(k+1-j, k-j)}\right]\left[\begin{array}{l}
J_{x m}^{(k-j)}\left(x^{\prime}\right) \\
J_{z m}^{(k-j)}\left(x^{\prime}\right)
\end{array}\right]
\end{aligned}
$$

By equating (11) and (12), the coefficients at the top and bottom layers are in terms of all the current densities on the strips as shown in

$$
\begin{aligned}
{\left[\begin{array}{c}
a_{m}^{e(N)} \\
a_{m}^{h(N)} \\
a_{m}^{e(1)} \\
a_{m}^{h(1)}
\end{array}\right]=} & {\left[\prod_{i=k}^{N-1}\left[V^{i, i+1}\right] \mid \prod_{i=1}^{k-1}\left[\Lambda^{(k+1-i, k-i)}\right]\right]_{4 M \times 2 M}^{-1} } \\
& \times\left(\left[I^{k, k+1}\right]_{(4 M \times 2 M)}\left[\begin{array}{c}
J_{x m}^{(k)}\left(x^{\prime}\right) \\
J_{z m}^{(k)}\left(x^{\prime}\right)
\end{array}\right]\right. \\
& -\left[I^{(k, k-1)}\right]_{(4 M \times 2 M)}\left[\begin{array}{l}
J_{x m}^{(k-1)}\left(x^{\prime}\right) \\
J_{z m}^{(k-1)}\left(x^{\prime}\right)
\end{array}\right] \\
& +\sum_{j=k+1}^{N-1} \prod_{i=k+1}^{j}\left[V^{(i-1, i)}\right]\left[I^{(j, j+1)}\right]\left[\begin{array}{l}
J_{x m}^{(j)}\left(x^{\prime}\right) \\
J_{z m}^{(j)}\left(x^{\prime}\right)
\end{array}\right] \\
& -\sum_{j=2}^{k-1} \prod_{i=1}^{j-1}\left[\Lambda^{(k+1-i, k-i)}\right] \\
& \left.\times\left[I^{(k+1-j, k-j)}\right]\left[\begin{array}{l}
J_{x m}^{(k-j)}\left(x^{\prime}\right) \\
J_{z m}^{(k-j)}\left(x^{\prime}\right)
\end{array}\right]\right) .
\end{aligned}
$$

When all the current densities are known, all the coefficients at various layers can be readily obtained by the use of ( $9 a$ ), (9b), and (13).

Consequently, the tangential electric fields as shown in (6) at the arbitrary region $k$ can be written symbolically as

$$
\left[\begin{array}{l}
E_{x}^{(k)}\left(x, y \mid x^{\prime}\right) \\
E_{z}^{(k)}\left(x, y \mid x^{\prime}\right)
\end{array}\right]=\sum_{i=1}^{N-1}\left[Z^{(k, i)}(x, y)\right]^{\prime}\left[\begin{array}{l}
J_{x m}^{(i)}\left(x^{\prime}\right) \\
J_{z m}^{(i)}\left(x^{\prime}\right)
\end{array}\right] .
$$

By using of (10a) and (10b), (14a) can be rewritten as

$$
\left[\begin{array}{l}
E_{x}^{(k)}\left(x, y \mid x^{\prime}\right) \\
E_{z}^{(k)}\left(x, y \mid x^{\prime}\right)
\end{array}\right]=\sum_{i=1}^{N-1}\left[Z^{(k, i)}\left(x, y \mid x^{\prime}\right)\right]\left[\begin{array}{l}
J_{x}^{(i)}\left(x^{\prime}\right) \\
J_{z}^{(i)}\left(x^{\prime}\right)
\end{array}\right] .
$$

Notice that all the coefficients in (6) are now in terms of currents densities, where $\left[Z^{(k, i)}\right]$ is the dyadic Green's impedance function in the region $k$ as a result of all current densities $k=1,2, \ldots, N$ and $i=1,2, \ldots, N-1$.

Integrating (14a) along all the metal strips, one obtains general Green's impedance function equations where the unknowns are the current distributions on the metal strips and no longer the point sources

$$
\left[\begin{array}{l}
E_{x}^{(k)}(x, y) \\
E_{z}^{(k)}(x, y)
\end{array}\right]=\sum_{i=1}^{N-1}\left[Z^{(k, i)}(x, y)\right]^{\prime}\left[\begin{array}{c}
\tilde{J}_{x m}^{(i)} \\
\tilde{J}_{z m}^{(i)}
\end{array}\right]
$$

where (16) and (17), (see bottom of the page). Next, the surface current densities $J_{x}^{(i)}$ and $J_{z}^{(i)}$ are expanded in terms of a finite series by using the appropriate set of basis functions that will be discussed in the next section.

Followed by the use of Galerkin's procedure [29], [30], the final set of boundary conditions, (7e) and (7f), are satisfied. This results in a nonstandard eigenvalue equation, i.e.,

$$
\left[G_{q \times q}(\gamma)\right][x]=[0],
$$

where current coefficient vector $[x]$ contains all the unknown current coefficients of the metal strips. The integer subscript $q$ stands for the order of the square matrix $[G]$. The nontrivial solution of (18) mandates that

$$
\operatorname{det}\left[G_{q \times q}(\gamma)\right]=0 .
$$

Once the propagation constants $(\gamma)$ is obtained the unknown coefficients of basis functions can be readily solved. Given the solutions for all the current distributions on the microstrips and slotlines, the electromagnetic fields inside the generalized waveguide can be computed by (9a), (9b), (13), and (4)-(6).

It is interesting to observe that when all the substrates are layered, the above mentioned equations will be reduced to the well-known SDA formulation. Such results are documented in Appendix B for reference.

$$
\begin{aligned}
& \tilde{J}_{x m}^{(i)}=\int_{y=d i} J_{x m}^{(i)} d x^{\prime}=
\end{aligned}
$$

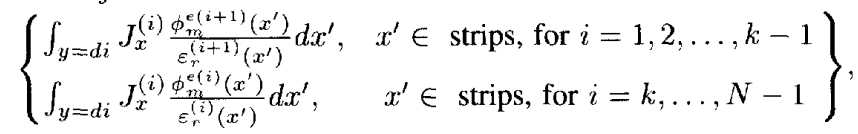

$$
\begin{aligned}
& \tilde{J}_{z m}^{(i)}=\int_{y=d i} J_{z m}^{(i)} d x^{\prime}= \\
& \left\{\begin{array}{l}
\int_{y=d i} J_{z}^{(i)} \phi_{m}^{h(i+1)}\left(x^{\prime}\right) d x^{\prime}, \quad x^{\prime} \in \text { strips, for } i=1,2, \ldots, k-1 \\
\int_{y=d i} J_{z}^{(i)} \phi_{m}^{h(i)}\left(x^{\prime}\right) d x^{\prime}, \quad x^{\prime} \in \text { strips, for } i=k, \ldots, N-1
\end{array}\right\} .
\end{aligned}
$$




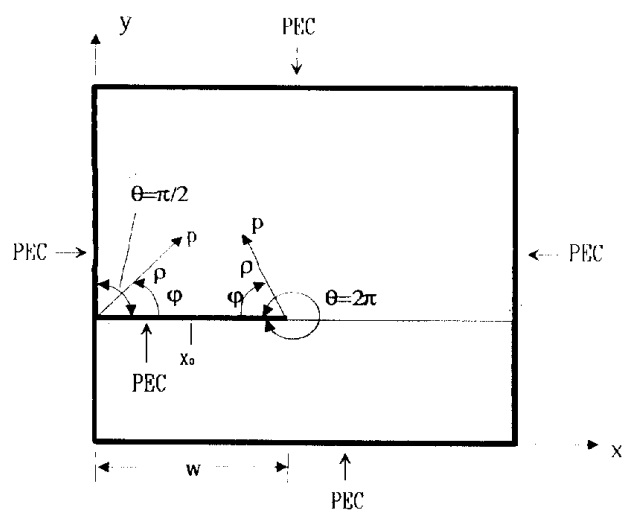

Fig. 2. Illustration of two edge conditions of a metallic strip: $\theta=\pi / 2$ and $\theta=2 \pi$.

\section{NeW Global Basis FunCtion fOR SLOTINE PROBlemS USING GREEN'S IMPENDANCE FUNCTION APPROACH}

To solve the Green's impedance function equations (15) by the Galerkin's procedure, the unknown surface currents on the microstrip type and slotline type transmission lines will be expressed by a known set of basis functions with unknown coefficients prefix. The most important attribute of the basis functions, when they are properly determined, is that the unknown current distributions of the strips can converge to nearly true current distributions. A few examples of such phenomena had been reported for the nearly true current distributions of the suspended coupled microstrip lines in the case of complex modes [26] and dominant modes [31], respectively. Thereby such preconditioned basis functions are global in nature and they will result in a matrix $G_{q \times q}(\gamma)$ in (18) of fairly small dimension, since $q$ is directly related to the number of the basis functions.

Refer to Fig. 2, which shows perfect conductor strip of width $w$ having one side connected to the side-wall which is also a perfect electric conductor (PEC). The strip contains two kinds of edge conditions, i.e., 1) $\theta=2 \pi$ at the one side, and 2) $\theta=\pi / 2$ at the other side. From the twodimensional electrostatic wedge problem, one may obtain a complete solution of surface charge distribution near end points $\left.p\right|_{\varphi=0}$ [32]

$$
\sigma(\rho)=\sum_{n=1}^{\infty} a_{n}^{\prime} \rho^{\left(\frac{n \pi}{\theta}\right)-1},
$$

where $\rho$ is the distance measured from one of the two end points, and $a_{n}^{\prime}$ are constants.

Normalizing variable $\rho$ with respect to half of the strip width, the new variable $u$ can be defined as

$$
u=2\left(x-x_{0}\right) / w
$$

where $x_{0}$ denotes the center point of strip. Substituting (21) into (20) results in

$$
\sigma(u)=\sum_{n=1}^{\infty} a_{n}(1 \pm u)^{\frac{n \pi}{\theta}-1},
$$

where plus is for left side edge condition, and minus for right side edge condition. (22) will be used in the following context. In (22), the $a_{n}$ are unknown constants and $-1<u<1$.

For different edge conditions, we should select the proper bases, namely:

1) For $\theta=2 \pi$, the derivation of basis functions out of (22) had been reported in literature [26] and is summarized here for reference:

$$
\begin{aligned}
j_{z n}^{ \pm}(u, \theta=2 \pi)= & (1 \pm u)^{\frac{n}{2}-1}-\sqrt{2}(1 \pm u)^{\frac{n}{2}-\frac{1}{2}} \\
& +\frac{1}{2}(1 \pm u)^{\frac{n}{2}} \\
j_{x n}^{ \pm}(u, \theta=2 \pi)= & (1 \pm u)^{\frac{n}{2}}-\sqrt{2}(1 \pm u)^{\frac{n}{2}+\frac{1}{2}} \\
& +\frac{1}{2}(1 \pm u)^{\frac{n}{2}+1}
\end{aligned}
$$

where $n$ is the order of the expansion functions; $n=$ $1,2, \ldots, N_{b}$.

2) The expansion functions near the edge $(\theta=\pi / 2)$ can be derived as follows. Since the longitudinal strip current $J_{z}$ is proportional to the charge distribution $\sigma(x)$ denoted by (22) when $\theta=\pi / 2$ and $x=0$ (or $u=-1$ ) as shown in Fig. 2 , the $n_{\mathrm{th}}$ order basis $j_{z n}^{+}$with current vanishing at $x=w(u=1)$, can be expressed as

$$
\begin{aligned}
j_{z n}^{+}\left(u, \theta=\frac{\pi}{2}\right)= & (1+u)^{2 n-1}-\frac{1}{2}(1+u)^{2 n+1} \\
& +\frac{1}{16}(1+u)^{2 n+3}, \quad \text { for } n=1,2, \ldots, N_{b} .
\end{aligned}
$$

Similarly, when the metal strip is connected to side-wall at $x=w(u=1)$ with the opposing end open, $j_{z n}^{-}$is

$$
\begin{aligned}
j_{z n}^{-}\left(u, \theta=\frac{\pi}{2}\right)= & (1-u)^{2 n-1}-\frac{1}{2}(1-u)^{2 n+1} \\
& +\frac{1}{16}(1-u)^{2 n+3}, \text { for } n=1,2, \ldots, N_{b} .
\end{aligned}
$$

It is interesting to observe that when $\rho(u)$ approaches $0( \pm 1), j_{z n}$ has $\rho^{1}$ asymptote.

By integrating the following equation imposed on the metal strip as was done in [33],

$$
\frac{\partial}{\partial x}\left(j_{x n}^{ \pm}(x) e^{-j \gamma z}\right)+\frac{\partial}{\partial z}\left(j_{z n}^{ \pm}(x) e^{-j \gamma z}\right)=j \omega \sigma(x) e^{-j \gamma z}
$$

where $\sigma(x)$ has been defined in (20). It follows that $j_{x n}^{ \pm}$is an integration of $j_{z n}^{ \pm}$. Therefore,

$$
\begin{aligned}
j_{x n}^{ \pm}\left(u, \theta=\frac{\pi}{2}\right)= & (1 \pm u)^{2 n}-\frac{1}{2}(1 \pm u)^{2 n+2} \\
& +\frac{1}{16}(1 \pm u)^{2 n+4}, \text { for } n=0,1,2, \ldots N_{b} .
\end{aligned}
$$

Notice that the index $n$ of $j_{x n}^{ \pm}\left(u, \theta=\frac{\pi}{2}\right)$ starts from 0 not 1. By doing so, the transverse current distribution $J_{x}(u)$ near edge will contain d.c. component. 
In summary, the basis functions for microstrip problems are

$$
\begin{aligned}
J_{z}(u)= & \sum_{n=1}^{N_{b}} c_{n}^{+} j_{z n}^{+}(u, \theta=2 \pi) \\
& +\sum_{n=1}^{N_{b}} c_{n}^{-} j_{z n}^{-}(u, \theta=2 \pi), \\
J_{x}(u)= & \sum_{n=1}^{N_{b}} d_{n}^{+} j_{x n}^{+}(u, \theta=2 \pi) \\
& +\sum_{n=1}^{N_{b}} d_{n}^{-} j_{x n}^{-}(u, \theta=2 \pi),
\end{aligned}
$$

and for slotline problems are

$$
\begin{aligned}
J_{z}(u)= & \sum_{n=1}^{N_{b}} c_{n}^{+} j_{z n}^{+}\left(u, \theta=\frac{\pi}{2}\right) \\
& +\sum_{n=1}^{N_{b}} c_{n}^{-} j_{z n}^{-}(u, \theta=2 \pi), \\
J_{x}(u)= & \sum_{n=0}^{N_{b}} d_{n}^{+} j_{x n}^{+}\left(u, \theta=\frac{\pi}{2}\right) \\
& +\sum_{n=1}^{N_{b}} d_{n}^{-} j_{x n}^{-}(u, \theta=2 \pi),
\end{aligned}
$$

where $c_{n}^{ \pm}$and $d_{n}^{ \pm}$are unknown coefficients; $N_{b}$ is the order of the $J_{x}$ and $J_{z}$ basis functions in the expansion.

\section{VALIDITY CHECK}

To validate our formulations presented in Sections II and III, this section will conduct the convergence study and then compare the results obtained by the present formulations with existing literature.

\section{A. Convergence Study}

The dominant mode propagation constant and its corresponding characteristic impedance is of primary concern in our study. These parameters are function of the order of basis function $N_{b}$ and the number of eigenfunction expansion terms $M(5),(27)$, and (28). Fig. 3(a) and (b) illustrates the convergent properties of the normalized propagation constant $\gamma / k_{0}$ and characteristic impedance $Z_{0}$ with respect to $M$ using $N_{b}$ as the controlling parameter, respectively. The global basis functions of (27a) and (27b) are incorporated in the numerical investigation. The characteristic impedance is given by the power-current definition

$$
Z_{0}=\frac{2 P}{I^{2}}
$$

where

$$
I=\int_{w} J_{z} d x
$$

and the transported power is calculated as

$$
P=\frac{1}{2} \operatorname{Re} \int_{s_{g}} \vec{E} \times \vec{H}^{*} \bullet d \vec{s}
$$

where $s_{g}$ is the whole cross-sectional area of the guide.

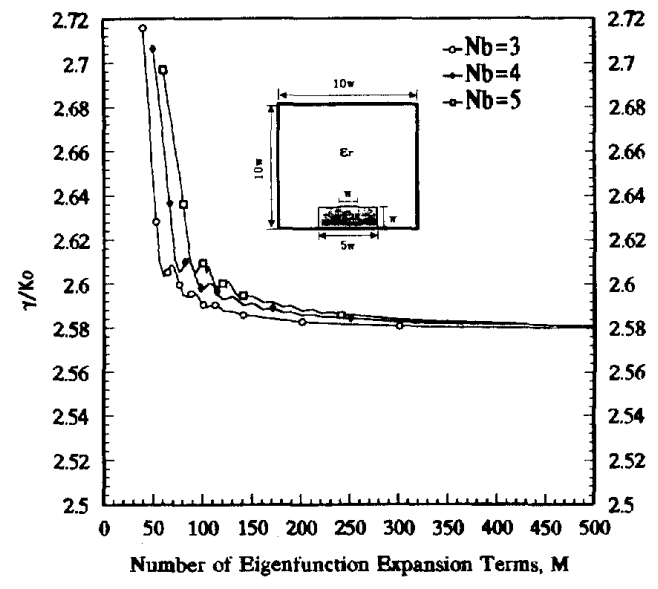

(a)

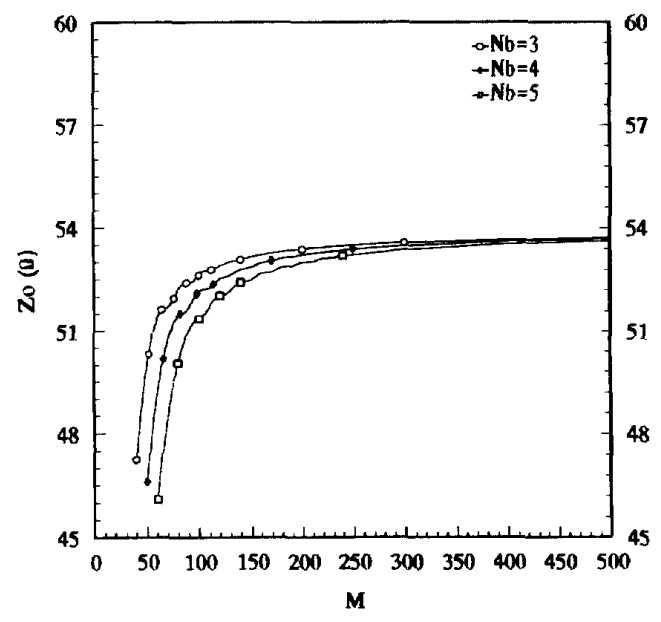

(b)

Fig. 3. Convergence behavior of the propagation characteristics of the fundamental mode for a shielded symmetric microstrip line at $10 \mathrm{GHz}$, when varying the number of basis function terms $\left(N_{b}\right)$ and the number of eigenfunction expansion terms $(M)$. Structural parameters are $w=1.27 \mathrm{~mm}, c_{12}=8.875$, and $\varepsilon_{r}=1.0$. (a) The normalized propagation constant. (b) The characteristic impedance.

In the particular case study of Fig. 3, where both structural parameters and material constants are given, when $N_{b}=3$ and $M=200,0.39 \%$ deviation is observed for $\gamma / k_{0}$ and $Z_{0}$ with respect to those obtained by using $N_{b}=5$ and $M=500$, respectively. Our experiences of using the new program indicate that for most commonly used planar and quasi-planar structures, the condition that $N_{b}=3$ and $M=250$ is sufficient for very good solution. Similar conclusion was also reported in [34].

\section{B. Validity Check: Comparison of New Results With Existing Literature}

To validate our program based on the new formulations, the modified Microslab, the microstrip proximity effect, and the slotline are used as three test cases for the comparative study against the data available in existing literature [22], [23], [25]. 


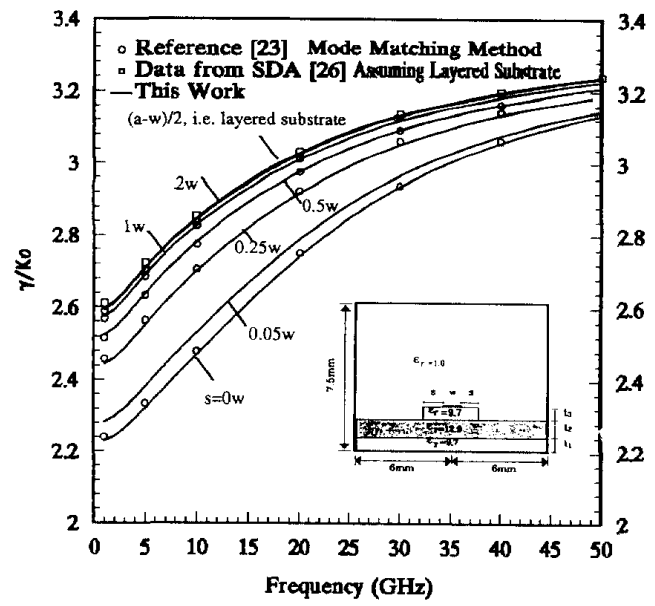

(a)

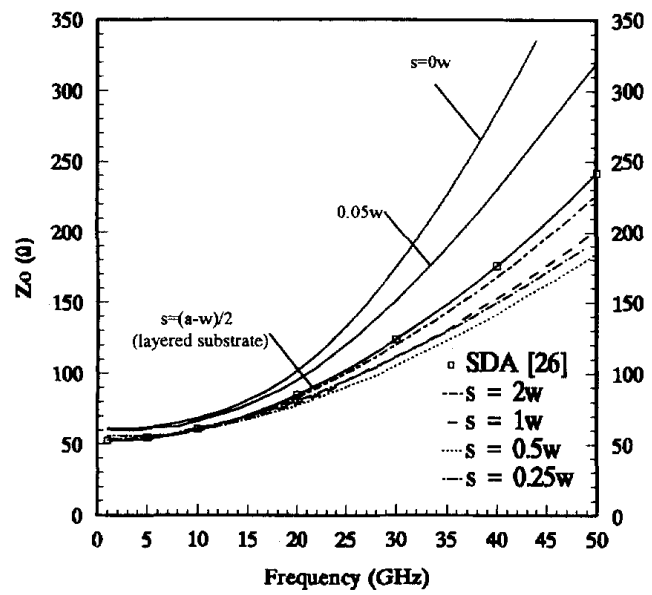

(b)

Fig. 4. Proximity effects of the extended supporting dielectric substrate $\left(\varepsilon_{r}=9.7\right)$ on (a) the dominant mode normalized propagation constant and (b) the characteristic impedance of a shielded symmetric modified Microslab when varying the value of $s$. Structural parameters are $w=1.2 \mathrm{~mm}, t_{1}=0.48 \mathrm{~mm}$, $t_{2}=0.71 \mathrm{~mm}, a=12.0 \mathrm{~mm}$, and $t_{3}=0.48 \mathrm{~mm}$. $N_{b}=3$, and $M=100$.

Given the modified Microslab as shown in Fig. 4, parts (a) and (b) show the normalized propagation constant $\left(\gamma / k_{0}\right)$ and the characteristic impedance $Z_{0}$, respectively. The solid and dashed lines are the data obtained by the present formulations using $N_{b}=3$ and $M=100$ under various conditions that the parameter $s$ changes from zero to $(a-w) / 2$. The change of $s$ from zero to $(a-w) / 2$ implies that the transmission line undergoes a transformation from the nonlayered Microslab to the modified Microslab and finally the layered microstrip structure. The corresponding solutions obtained by the SDA program [26] assuming the layered structure are denoted by the square symbols, whereas those obtained by the mode-matching method [23] are marked by the circle symbols. In all cases excellent agreement is obtained.

Fig. 5 shows the results for the second case study where the numerical results obtained by present formulation are

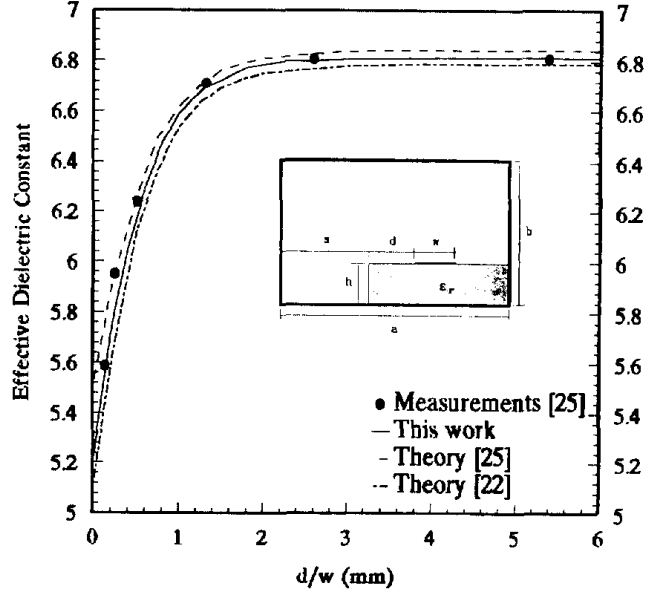

Fig. 5. Comparison of this work to [22], [25], in the calculation of effective dielectric constant of a microstrip as a function of $d / w$, the normalized distance between the edge of substrate and that of microstrip. Structural parameters are $\varepsilon_{r}=10.2, w=0.95 \mathrm{~mm}, h=1.27 \mathrm{~mm}, a=50.0 \mathrm{~mm}$, $b=35.0 \mathrm{~mm}, s=15.0 \mathrm{~mm}$, and frequency $=2 \mathrm{GHz}$

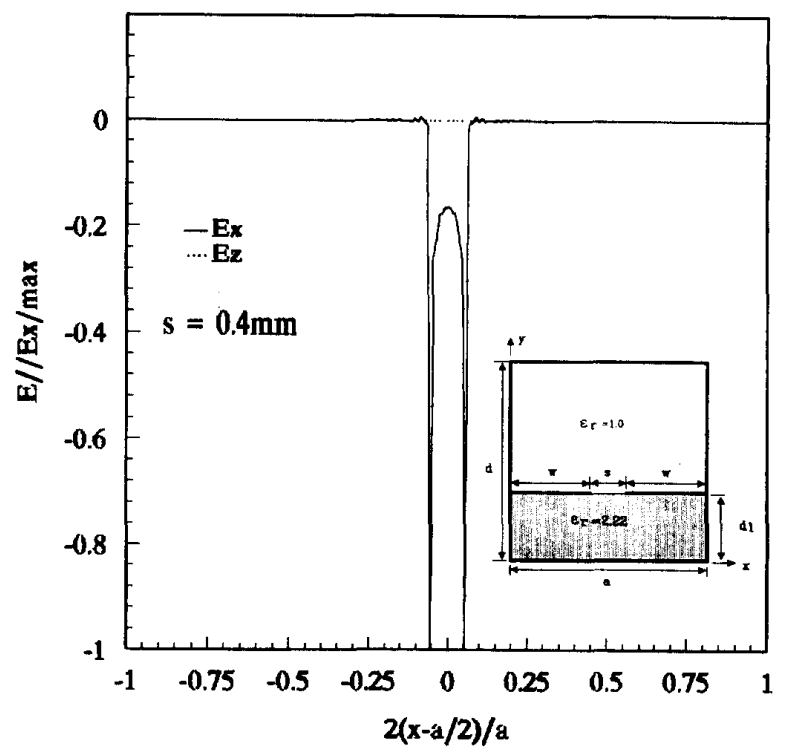

Fig. 6. The normalized tangential $E$-field components of the dominant mode at $y=d_{1}, f=30 \mathrm{HGz}$. The insert shows the structure of the symmetric conductor-backed slotline. Structural parameters are $a=7.112 \mathrm{~mm}$, $d_{1}=3.556 \mathrm{~mm}, \boldsymbol{d}=10.668 \mathrm{~mm}$, and $s=0.4 \mathrm{~mm}$.

compared with those reported in [22], [25]. Again very good agreement is achieved between our work and the theoretic results and measurement [22], [25].

The last case study is to investigate the slotline problem using the Green's impedance function and the new basis functions (28a) and (28b). Similar convergence study to those reported in Fig. 3(a) and (b) had been conducted. Fig. 6 displays the tangential $E$-field components of slotline directly at the interface plane, $y=d_{1}$, at $30 \mathrm{GHz}$. In order to obtain the 


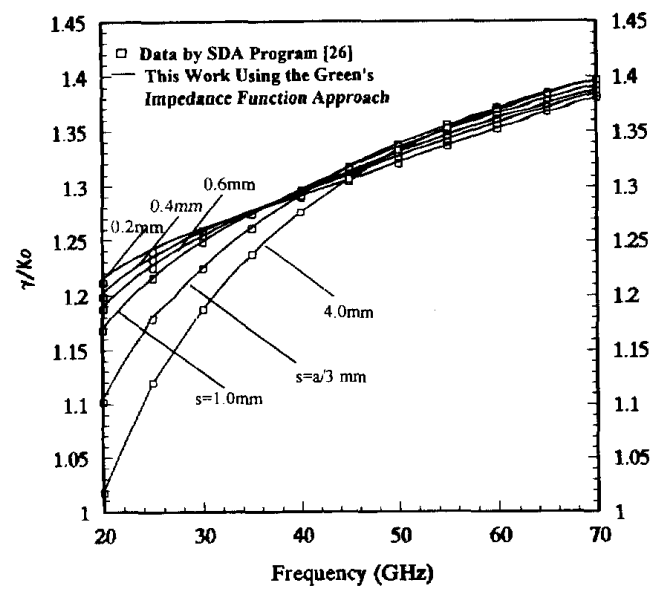

(a)

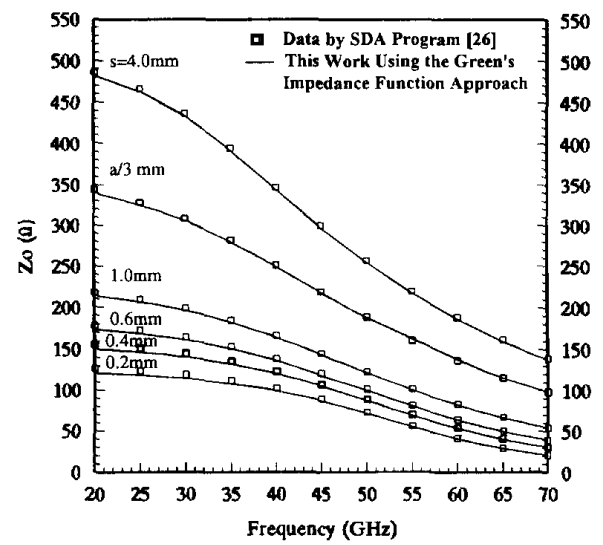

(b)

Fig. 7. Comparison of the propagation characteristics of the dominant mode of the structure illustrated as an inset in Fig. 6 when varying the value of $s$ Results denoted by rectangular symbols are based on SDA program [26] using the slot field expansions. Results from this work using the Green's impedance approach are indicated by solid lines. (a) The normalized propagation constant. (b) The characteristic impedance. When $N_{b}=5$ and $M=240$.

very accurate tangential $E$-field components at the interface, the condition that $N_{b}=5$ and $M=5 \times 10^{3}$ is necessary. The tangential $E$-field are normalized by the maximum $E_{x}$-field $\left(E_{x \mid \text { max }}\right)$. Fig. 6 shows that both tangential fields $E_{z}$ and $E_{x}$ vanish along the metal strips and the $\rho^{-1 / 2}$, singularity clearly exists for $E_{x}$ near the ends of the slotline.

By applying the power-voltage definition for slotline characteristic impedance, i.e.,

$$
Z_{0}=\frac{\left|V_{s}\right|^{2}}{2 P}
$$

where $P$ is the transported power associated with slot as $(29 \mathrm{c})$ and $V_{s}$ is the voltage across the slot given by

$$
V_{s}=\int_{s} E_{x}\left(x, d_{k}\right) d x
$$

Fig. 7(a) and (b) displays the normalized propagation constant and the characteristic impedance between $20 \mathrm{GHz}$ and $70 \mathrm{GHz}$ using the slot width $s$ as the controlling variable. Our results and those obtained by the SDA program [26] with the basis functions represent the electric field distribution in the slot are represented by solid lines and square symbols, respectively. Only when in the low frequency limit near $20 \mathrm{GHz}$ and narrow slot $(a / s=35.56)$, the particular case study shows that about $3.5 \%$ deviation between the new formulation and the SDA program [26] for the characteristic impedance shown in the lower left corner of the Fig. 7(b). The deviation of the propagation constant, however, is very slight and only $0.33 \%$ is observed for the same condition. Further investigations have shown that for all normally used slotlwidths, a five-term expansion functions according to (28a) and (28b) and a number of 250 eigenfunction expansion terms are sufficient for very good solutions.

\section{CONCLUSION}

This paper proposes and validates a full-wave integral equation technique based on the Green's impedance function approach and newly derived basis functions for obtaining the dispersion characteristics of a system of strip lines and slotlines integrated on multiple nonlayered dielectric substrates. The recursive formulation of such a complicated and generic problem makes the derivation of Green's impedance function systematic and easy to implement. To solve the slotline type problem using the Green's impedance function a new set of basis functions is derived to expand the unknown current distributions on the metal strips of the slotline. The use of the resultant integral equation incorporating the Green's impedance function and the new basis functions is confirmed by conducting both convergence study and the validity check against existing literature, where three examples are invoked, namely, Microslab, microstrip proximity effect and conductorbacked slotline. Excellent agreement for the propagation characteristics obtained by the present method and all the three case studies is obtained.

\section{APPENDIX A}

The upper chain matrix $\left[V^{(k, k+1)}\right]$ and the current matrix $\left[I^{(k, k+1)}\right]$ in $(9 \mathrm{a})$ are given by

$$
\begin{aligned}
{\left[V^{(k, k+1)}\right] } & =\left[\begin{array}{llll}
V_{11} & V_{12} & V_{13} & V_{14} \\
V_{21} & V_{22} & V_{23} & V_{24} \\
V_{31} & V_{32} & V_{33} & V_{34} \\
V_{41} & V_{42} & V_{43} & V_{44}
\end{array}\right], \\
{\left[I^{(k, k+1)}\right] } & =\left[\begin{array}{ll}
I_{11} & I_{12} \\
I_{21} & I_{22} \\
I_{31} & I_{32} \\
I_{41} & I_{42}
\end{array}\right]
\end{aligned}
$$

where

$$
V_{11}(m, n)=\frac{1}{Q_{m}}\left[\frac{Z_{e e}^{4}}{\tan \left(\beta_{y m}^{e(k)} y_{k}^{\prime}\right) \tan \left(\beta_{y n}^{e(k+1)} y_{k+1}^{\prime}\right)}-Z_{c e}^{1}\right],
$$




$$
\begin{aligned}
& V_{12}(m, n)=\frac{1}{Q_{m}}\left[\frac{Z_{e e}^{4} \tan \left(\beta_{y n}^{e(k+1)} y_{k+1}^{\prime}\right)}{\tan \left(\beta_{y m}^{e(k)} y_{k}^{\prime}\right)}+Z_{e e}^{1}\right] \\
& V_{13}(m, n)=-V_{14}(m, n)=\frac{1}{Q_{m}}\left[\frac{-Z_{\mathrm{eh}}^{4}}{\tan \left(\beta_{y m}^{e(k)} y_{k}^{\prime}\right)}\right] \text {, } \\
& V_{21}(m, n)=\frac{-1}{P_{m}}\left[\frac{Z_{e e}^{4} \tan \left(\beta_{y m}^{e(k)} y_{k}^{\prime}\right)}{\tan \left(\beta_{y n}^{e(k+1)} y_{k+1}^{\prime}\right)}+Z_{e e}^{1}\right] \text {, } \\
& V_{22}(m, n)=\frac{-1}{P_{m}}\left[\tan \left(\beta_{y m}^{e(k)} y_{k}^{\prime}\right) \tan \left(\beta_{y n}^{e(k+1)} y_{k+1}^{\prime}\right) Z_{e e}^{4}-Z_{e e}^{1}\right] \text {, } \\
& V_{23}(m, n)=-V_{24}(m, n)=\frac{1}{P_{m}}\left[\tan \left(\beta_{y m}^{e(k)} y_{k}^{\prime}\right) Z_{e h}^{4}\right], \\
& V_{31}(m, n)=-V_{32}(m, n)=\frac{1}{P_{m}}\left[\tan \left(\beta_{y m}^{h(k)} y_{k}^{\prime}\right) Z_{h \mathrm{e}}^{2}\right], \\
& V_{33}(m, n)=\frac{-1}{P_{m}}\left[\tan \left(\beta_{y m}^{h(k)} y_{k}^{\prime}\right) \tan \left(\beta_{y n}^{h(k+1)} y_{k+1}^{\prime}\right) Z_{h h}^{2}-Z_{h h}^{3}\right], \\
& V_{34}(m, n)=\frac{-1}{P_{m}}\left[\frac{Z_{h h}^{2} \tan \left(\beta_{y m}^{h(k)} y_{k}^{\prime}\right)}{\tan \left(\beta_{y n}^{h(k+1)} y_{k+1}^{\prime}\right)}-Z_{h h}^{3}\right], \\
& V_{42}(m, n)=-V_{41}(m, n)=\frac{-1}{Q_{m}}\left[\frac{Z_{h e}^{2}}{\left.\tan \beta_{y m}^{h(k)} y_{k}^{\prime}\right)}\right] \text {, } \\
& V_{43}(m, n)=\frac{1}{Q_{m}}\left[\frac{Z_{h h}^{2} \tan \left(\beta_{y m}^{h(k)} y_{k}^{\prime}\right)}{\tan \left(\beta_{y n}^{h(k+1)} y_{k+1}^{\prime}\right)}+Z_{h h}^{3}\right] \text {, } \\
& V_{44}(m, n)=\frac{1}{Q_{m}}\left[\frac{Z_{h h}^{2}}{\tan \left(\beta_{y m}^{y(k)} y_{k}^{\prime}\right) \tan \left(\beta_{y n}^{h(k+1)} y_{k+1}^{\prime}\right)}+Z_{h h}^{3}\right] \text {, } \\
& I_{11}(m, n)=\frac{1}{Q_{m}}\left[\frac{P_{x 1}}{\tan \left(\beta_{y m}^{e(k)} y_{k}^{\prime}\right)}\right] \text {, } \\
& Z_{h e}^{2}=\frac{\gamma}{\omega \mu_{\mathrm{o}} \beta_{y m}^{h(k)} \delta_{m n}^{h}}\left[Z_{e e}^{1} \int \frac{1}{\varepsilon_{r}^{(k)}(x)} \frac{\partial \phi_{n}^{e(k)}(x)}{\partial x} \phi_{m}^{h(k)}(x) d x\right. \\
& \left.-\int \frac{1}{\varepsilon_{r}^{(k+1)}(x)} \frac{\partial \phi_{n}^{e(k+1)}(x)}{\partial x} \phi_{m}^{h(k)}(x) d x\right] \text {, } \\
& Z_{h h}^{2}=\frac{\beta_{y n}^{h(k+1)}}{\beta_{y m}^{h(k)}} \frac{\int \phi_{n}^{h(k+1)}(x) \phi_{m}^{h(k)}(x) d x}{\delta_{m n}^{h}}, \\
& Z_{h h}^{3}=\frac{\left[\left(\beta_{y n}^{h(k+1)}\right)^{2}+\gamma^{2}\right]}{\left[\left(\beta_{y m}^{h(k)}\right)^{2}+\gamma^{2}\right]} \frac{\int \phi_{n}^{h(k+1)}(x) \phi_{m}^{h(k)}(x) d x}{\delta_{m n}^{h}}, \\
& Z_{\mathrm{ee}}^{4}=\frac{\beta_{y n}^{e(k+1)} \int \phi_{n}^{e(k+1)}(x) \frac{\phi_{n}^{\mathrm{e}(k)}(x)}{\varepsilon_{r}^{(k)}(x)} d x}{\beta_{y m}^{e(k)}} \frac{\delta_{m n}^{e}}{\varepsilon_{m}} \\
& Z_{e h}^{4}=\frac{\gamma}{\omega \varepsilon_{0} \beta_{y m}^{e(k)} \delta_{m n}^{e}}\left[Z_{h h}^{3} \int \frac{\phi_{m}^{e(k)}(x)}{\varepsilon_{r}^{(k)}(x)} \frac{\partial \phi_{n}^{h(k)}(x)}{\partial x} d x\right. \\
& \left.-\int \frac{\phi_{m}^{e(k)}(x)}{\varepsilon_{r}^{(k)}(x)} \frac{\partial \phi_{n}^{h(k+1)}}{\partial x} d x\right] \\
& \delta_{m n}^{e}=\int \frac{\phi_{m}^{e(k)}(x)}{\varepsilon_{r}^{(k)}(x)} \phi_{n}^{e(k)}(x) d x, \\
& \delta_{m n}^{h}=\int \phi_{m}^{h(k)}(x) \phi_{n}^{h(k)}(x) d x, \\
& P_{z 1}=\frac{1}{\left[\left(\beta_{y m}^{h(k)}\right)^{2}+\gamma^{2}\right] \delta_{m n}^{h}}, \\
& P_{z 2}=\frac{\gamma P_{z 1} \int \frac{\partial \phi_{n}^{h(k)}(x)}{\partial x} \frac{\phi_{m}^{e(k)}(x)}{\varepsilon_{r}^{(k)}(x)} d x}{\omega \varepsilon_{0} \beta_{y m}^{e(k)} \delta_{m n}^{e}}, \\
& P_{x 1}=\frac{1}{j \omega \varepsilon_{0} \beta_{y m}^{e(k)} \delta_{m n}^{e}}
\end{aligned}
$$$$
I_{12}(m, n)=\frac{-1}{Q_{m}}\left[\frac{P_{z 2}}{\tan \left(\beta_{y m}^{e(k)} y_{k}^{\prime}\right)}\right] \text {, }
$$$$
I_{21}(m, n)=\frac{-1}{P_{m}}\left[\tan \left(\beta_{y m}^{e(k)} y_{k}^{\prime}\right) P_{x 1}\right] \text {, }
$$$$
I_{22}(m, n)=\frac{1}{P_{m}}\left[\tan \left(\beta_{y m}^{e(k)} y_{k}^{\prime}\right) P_{z 2}\right],
$$$$
I_{31}(m, n)=I_{41}(m, n)=0 \text {, }
$$$$
I_{22}(m, n)=\frac{1}{P_{m}} P_{z 1} \text {, }
$$$$
I_{22}(m, n)=\frac{1}{Q_{m}} P_{z 1}
$$

and

$$
\begin{aligned}
P_{m} & =1+\frac{1}{\tan ^{2}\left(\beta_{y m}^{e(k)} y_{k}^{\prime}\right)}, \\
Q_{m} & =1+\tan ^{2}\left(\beta_{y m}^{e(k)} y_{k}^{\prime}\right), \\
Z_{e e}^{1} & =\frac{\left[\left(\beta_{y n}^{e(k+1)}\right)^{2}+\gamma^{2}\right]}{\left[\left(\beta_{y m}^{e(k)}\right)^{2}+\gamma^{2}\right]} \cdot \frac{\int \frac{\phi_{n}^{e(k+1)}(x)}{\varepsilon_{r}^{(k+1)}(x)} \phi_{m}^{e(k)}(x) d x}{\delta_{m n}^{e}}
\end{aligned}
$$

where $\beta_{y m}^{e(k)}, \beta_{y n}^{e(k+1)}, \beta_{y m}^{h(k)}, \beta_{y n}^{h(k+1)}, y_{k}^{\prime}$, and $y_{k+1}^{\prime}$ are given in $(5 \mathrm{c})$.

Similarly, by the same mathematical manipulations and proper change of the indices and symbols, the lower chain matrix and the current matrix in (9b) can be obtained by using the procedures described in the above.

\section{APPENDIX B}

The Fourier sine and cosine transforms in the closed structure can be defined as

$$
\begin{aligned}
& F(\alpha)=\int_{0}^{a} f(x) \sin (\alpha x) d x \\
& F(\alpha)=\int_{0}^{a} f(x) \cos (\alpha x) d x, \text { respectively. }
\end{aligned}
$$

In Section II-A, we describe the proposed generalized fullwave integral equation and derive the general dyadic Green's impedance function. First, in order to solve those unknown coefficients, we apply the boundary condition 7 (a) to 7(d) at the interface by multiplying both sides by $\phi_{m}^{e(k)}(x)$ and $\phi_{m}^{h(k)}(x)$, respectively, and integrating from 0 to $a$, to obtain the relating equations (9a) and (9b). Second, after some algebraic manipulations, we obtain the dyadic Green's impedance 
function using the point current densities as unknowns. Then by integrating over the metal strips, we can obtain the generic dyadic Green's impedance function governed by arbitrary current distributions as shown in (15).

Now if every dielectric substrate is layered in configuration, the potential functon $\phi_{m}^{e(k)}(x)$ and $\phi_{m}^{h(k)}(x)$ can be expressed as

$$
\begin{aligned}
\phi_{m}^{e(k)}(x) & =\cos \left(\frac{m \pi}{a} x\right), & & k=1,2, \ldots, N \\
\phi_{m}^{h(k)}(x) & =\sin \left(\frac{m \pi}{a} x\right), & k & =1,2, \ldots, N
\end{aligned}
$$

Thus the integrals of the boundary condition (7a)-(7d) can be rewritten as

$$
\begin{aligned}
& \int E_{z}^{(k+1)} \sin \left(\frac{m \pi}{a} x\right) d x-\int E_{z}^{(k)} \sin \left(\frac{m \pi}{a} x\right) d x=0 \\
& \int H_{z}^{(k+1)} \cos \left(\frac{m \pi}{a} x\right) d x-\int H_{z}^{(k)} \cos \left(\frac{m \pi}{a} x\right) d x \\
& =J_{x}^{(k)} \cos \left(\frac{m \pi}{a} x^{\prime}\right) . \\
& \int E_{z}^{(k+1)} \cos \left(\frac{m \pi}{a} x\right) d x-\int E_{x}^{(k)} \cos \left(\frac{m \pi}{a} x\right) d x=0, \\
& \int H_{x}^{(k)} \sin \left(\frac{m \pi}{a} x\right) d x-\int H_{x}^{(k+1)} \sin \left(\frac{m \pi}{a} x\right) d x \\
& =J_{z}^{(k)} \sin \left(\frac{m \pi}{a} x^{\prime}\right) .
\end{aligned}
$$

Similarly, the Equations (17) and (18) are now rewritten as

$$
\begin{gathered}
\tilde{J}_{x m}^{(i)}=\int_{y=d_{i}} J_{x m}^{(i)} d x^{\prime}=\int_{y=d_{i}} J_{x}^{(i)} \cos \left(\frac{m \pi}{a} x^{\prime}\right) d x^{\prime}, \\
i=1,2, \ldots, N-1 \\
\tilde{J}_{z m}^{(i)}=\int_{y=d_{i}} J_{z m}^{(i)} d x^{\prime}=\int_{y=d_{i}} J_{z}^{(i)} \sin \left(\frac{m \pi}{a} x^{\prime}\right) d x^{\prime}, \\
i=1,2, \ldots, N-1 .
\end{gathered}
$$

According to the Fourier transforms defined in (B-1) and B2), (B-5)-(B-7) are now in Fourier transform domain. The SDA will lead to exactly the same expression sas shown in (B-5)-(B-7).

\section{ACKNOWLEDGMENT}

The authors express their appreciation to Prof. J.-D. Tseng and Prof. J.-T. Kuo for making their programs available for verification and comparison purposes.

\section{REFERENCES}

[1] K. Atsuki and E. Yamashita, "Transmission line aspects of the design of broad-band electrooptic traveling-wave modulators," Lightwave Tech., vol. LT-5. pp. 316-319, Mar. 1987

[2] E. Yamashita, K. Atsuki, and 'T. Akamatsu, "Application of microstrip analysis to design of a broad-band electrooptical modulator," IEEE Trans. Microwave Tech., vol. MTT-22, pp. 462-464, Apr. 1974.

[3] M. N. Khan, A. Gopinath, J. P, G. Bristow, and J. P. Donnelly, "Technique for velocity-matched traveling-wave electroptic modulator in $\mathrm{AlGaAs} / \mathrm{GaAs}$," IEEE Trans. Microwave Theory Tech., vol. 41, pp. 244-249, Feb. 1993.

[4] H. B. Sequeira and J. A. McClintock, "Microslab" waveguide for mm-wave frequencies," 5 th Benjamin Franklin Symp. Dig.. Philadelphia, PA, May 4, 1985. pp. 67-69.
[5] B. Young and T. Itoh, "Analysis and design of microslab waveguide," IEEE Trans. Microwave Theory Tech., vol. MTT-35, pp. 85(2-857, Sept. 1987.

[6] Y. S. Lee, "Mode compensation applied to parallel-coupled microstrip directional filter design," IEEE Trans. Microwave Theory Tech., vol. MTT-22, pp. 66-99, Jan. 1974

[7] B. Sheleg and B. E. Spielman, "Broad-band directional couplers using microstrip with dielectric overlays," IEEE Trans. Microwave Theory Tech., vol. MTT-22, pp. 1216-1220, Dec 1974.

[8] T. Itoh and J. Rivera, "Design of an overlay directional coupler by a fullwave analysis," IEEE Trans. Microwave Theorey Tech, vol. MTT-31, pp. 1017-1022, Dec. 1983.

[9] B. Roth, J. Borkes, M. Joseph, and A. Bayer, "A method for the development of monolithical integrated resonators on GiAs with increased Q-factor," in Proc. 20th European Microwave Conf. Budapest, Hungary, 1990, pp. 965-970.

[10] W.-T. Lo, C.-K. C. Tzuang, S.-T. Peng, C.-C. Chang, J.-W. Huang, and C.-C. Tien, "Resonant phenomena in conductor-backed coplanar waveguide (CBCPW)," IEEE MTT-S Int. Microwave Symp. Dig., June 1993, pp. 1199-1202.

[11] J. P. Webb, "Finite element analysis of dispersion in waveguides with sharp metal edges," IEEE Trans. Microwave Theory Tech., vol. MTT-36, pp. $1819-1824$, Dec. 1988.

[12] C.-K. C. Tzuang and T. Itoh, "Finite element analysis of slow wave Schottky contact printed lines," IEEE Trans. Microwave Theorv Tech. vol. MTT-34, pp. 1483-1489, Dec. 1986.

[13] M. Albani and P. Bernardi, "A Numerical method based on the discretization of Maxwell equations in integral form," IEEE Trans. Mi crowave Theory Tech., vol. MTT-22, pp. 446-450, Apr. 1974.

[14] K. Bierwirth, N. Schulz, and F. Arndt, "Finite-difference analysis of rectangular dielectric waveguide structures," IEEE Trans. Microwave Theory Tech., vol. MTT-34, pp. 1104-1114, Nov. 1986.

[15] D. H. Choi and W. J. R. Hoefer, "The finite-difference-time-domain method and its application to eigenvalue problems," IEEE Trans. Microwave Theory Tech., vol. MTT-34, pp. 1464-1470, Dec. 1986

[16] X. Zhang, J. Fang, and K. K. Mei, "Calculations of the dispersive characteristics of microstrips by the time-domain finite difference method," IEEE Trans. Microwave Theory Tech., vol. MTT-36. pp. 261-267, Feb. 1988.

[17] T. Itoh, Ed., Numerical Techniques for Microwave and Millimeter-Wave Passive Structures. New York: Wiley, 1989, chs. 3 and 5.

[18] G. Kowalsi and R. Pregla, "Dispersion characteristics of sheilded microstrips with finite thickness," Arch. Elek. Ubertragung., vol. 25 , pp. 193-196, Apr. 1971.

[19] J. B. Davies and D. Mirshekar-Syahkal, "Spectral domain solution of arbitrary coplanar transmission line with multilayer substrate," IEEE Trans. Microwave Theory Tech., vol. MTT-25, pp. 143-146, Feb. 1977.

[20] T. Itoh, "Spectral domain immitance approach for dispersion characteristics of generalized printed transmission lines," IEEE Trans. Microwave Tech, vol. MTT-28, pp. 733-736, July 1980.

[21] T. G. Livernois and L. P. B. Katehi, "A generalized method for deriving the space-domain Green's function in a shield, multilayer substrate structure with applications to MIS slow-wave transmission lines," IEEE Trans. Microwave Theory Tech., vol. 37, pp. 1761-1767, Nov. 1989.

[22] A. G. Engel, Jr. and L. P. B. Katehi, "Analysis of nicrostrip structures on and near dielectric ridge using an integral equation-mode matching tecnique,"IEEE MTT-s Int. Microwave Symp. Dig. 1991, pp. 135-138.

[23] C.-K. C. Tzuang and J. -D. Tseng, "A full-wave mixed potential modematching method for the analysis of planar or quasi-planar transmission lines," IEEE Trans. Microwave Theory Tech., vol. 39, pp. 1-11, Jan 1991.

[24] R. Pregla, M. Koch, and W. Pascher, "Analysis of hybrid waveguide structures consisting of microstrips and dielectric waveguides," in Proc 17th European Microwave Conf., Sept. 1987.

[25] M. Thorburn, A. Agoston, and V. K. Tripathi, "Computation of frequency-dependent propagation characteristics of microstriplike propagation structures with discontinuous layers," IEEE Trans Microwave Theory Tech., vol. 38, pp. 148-153, Feb. 1990.

[26] J.-T. Kuo and C.-K. C. Tzuang, "Complex modes in shielded suspended coupled microstrip lines," IEEE Trans. Microwave Theory Tech., vol. 38, pp. 1278-1286, Sept. 1990.

[27] W. Heinrich, "Full-wave analysis of conductor loss on MMIC transmission lines," IEEE Trans. Microwave Tech., vol. 38, pp. 1468-1472, Oct. 1990.

[28] C.-K. C. Tzuang, C.-D. Chen, and S. T. Peng, "Full-wave analysis of lossy quasi-planar transmission line incorporating the metal modes," IEEE Trans. Microwave Theory Tech., vol. 38, pp. 1792-1799, Dec, 1990. 
[29] R. F. Harrington, Time-Harmonic Electromagnetic Fields. New York: McGraw-Hill, 1961

[30] R. F. Harrington, "Matrix methods for field problems," Proc. IEEE, vol. 55 , pp. $136-149,1967$.

[31] C.-K. C. Tzuang and J.-T. Kuo, "Modal current distributions on closely coupled microstrip lines-A comparison study of the SDA basis functions," Electron. Lett., vol. 26, no. 7, pp. 464 465, Mar. 1990.

[32] J. D. Jackson, Classical Electrodynamics. New York: Wiley, 1975, pp. $75-78$.

[33] E. J. Denlinger, "A frequency dependent solution for microstrip transmission lines," IEEE Trans. Microwave Theory Tech., vol. MTT-19, pp. 30-39, Jan. 1971

[34] L.-P. Schnidt and T. Itoh, "Spectral domain analysis of dominant and higher order modes in fin-lines," IEEE Trans. Microwave Theory Tech., vol. MTT-28, pp. 981-985, Sept. 1980.

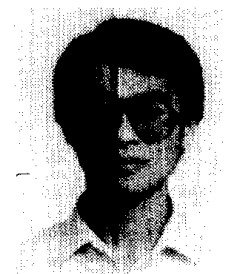

Jeng-Wen Huang (S'91) was born March 17, 1963, in Taiwan. He received the B.S. degree in electrical engineering from Feng Chia University, Taichung, Taiwan, and the M.S. and Ph.D. degrees in communication engineering from National Chiao Tung University, Hsinchu, Taiwan, in 1985, 1991, and 1994. respectively.

From 1987 to 1989 , he was a Teaching Assistant at the Shu-Te Institute of Technology. His research interests include microwave and millimeter-wave integrated circuit design.

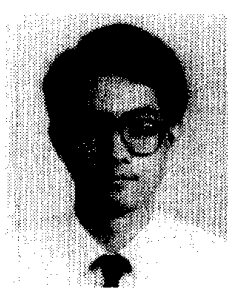

Ching-Kuang C. Tzuang (S'84-M'86-SM'92) was born May 10, 1955, in Taiwan. He received the B.S. degree in electric engineering from the National Chiao Tung University, Hsinchu, Taiwan the M.S. degree from the University of California at Los Angeles, and the Ph.D. degree from the University of Texas, Austin, in 1977, 1980, and 1986, respectively.

From February 1981 to June 1984 , he was with TRW, Redondo Beach, CA, working on analog and digital monolithic microwave integrated circuits.

Since September 1986, he has been with the Institute of Communication Engineering, National Chiao Tung University, Hsinchu, Taiwan. His research activities involve the design and development of millimeter-wave and microwave quasi-planar integrated circuits. 